LAWRENCE LIVERMORE N A T IO N A L LABORATORY

\title{
Chamber-transport simulation results for heavy-ion fusion drivers
}

W. M. Sharp, D. A. Callahan, M. Tabak, S. S. Yu, P. F. Peterson, D. V. Rose, D. R. Welch

October 21, 2004

Nuclear Fusion 
This document was prepared as an account of work sponsored by an agency of the United States Government. Neither the United States Government nor the University of California nor any of their employees, makes any warranty, express or implied, or assumes any legal liability or responsibility for the accuracy, completeness, or usefulness of any information, apparatus, product, or process disclosed, or represents that its use would not infringe privately owned rights. Reference herein to any specific commercial product, process, or service by trade name, trademark, manufacturer, or otherwise, does not necessarily constitute or imply its endorsement, recommendation, or favoring by the United States Government or the University of California. The views and opinions of authors expressed herein do not necessarily state or reflect those of the United States Government or the University of California, and shall not be used for advertising or product endorsement purposes. 


\title{
CHAMBER-TRANSPORT SIMULATION RESULTS FOR HEAVY-ION FUSION DRIVERS
}

\author{
W. M. Sharp, D. A. Callahan, and M. Tabak, \\ Lawrence Livermore National Laboratory, Livermore, CA 94550, USA \\ S. S. Yu \\ Lawrence Berkeley National Laboratory, Berkeley, CA 94720, USA \\ P. F. Peterson \\ University of California Berkeley, Berkeley, CA 94720, USA \\ D. V. Rose and D. R. Welch \\ Mission Research Corporation, Albuquerque, NM 87104, USA
}

\begin{abstract}
The heavy-ion fusion (HIF) community recently developed a power-plant design that meets the various requirements of accelerators, final focus, chamber transport, and targets. The point design is intended to minimize physics risk and is certainly not optimal for the cost of electricity. Recent chamber-transport simulations, however, indicate that changes in the beam ion species, the convergence angle, and the emittance might allow more-economical designs.
\end{abstract}

\section{INTRODUCTION}

The final transport of a heavy-ion beam to a fusion target poses conflicting demands. Analyses of powerplant economics strongly favor the use of ions with low energy and atomic mass, although this choice requires a higher total current on target, driving up either the number of beams or current per beam. The indirectdrive distributed-radiator targets ${ }^{1,2,3}$ presently preferred for heavy-ion fusion (HIF) require millimeter-sized beam-spot radii on the target ends. This requirement and the need for azimuthally symmetric deposition argue for using many beams, each with low emittance and net charge. Finally, the escape of radiation and neutrons from the target must be minimized to prolong magnet and fusion-chamber lifetimes. These objectives are met in HIF power-plant designs by using jets of molten salt to protect the fusion-chamber walls, although collisional ionization with the resulting background-gas in the chamber complicates beam transport to the target.

A point design meeting these constraints has recently been worked out. ${ }^{4}$ This so-called "robust point design" is deliberately conservative, aiming to minimizing the physics risk rather than the cost of electricity. A pivotal feature of the design is the use of low-density plasma in the beam line between the finalfocus magnets and the chamber to neutralize much of the beam space charge. ${ }^{5,67}$ Plasma electrons are trapped in the potential of the passing beam and are carried to the target, allowing substantial beam currents while still maintaining the low net charge needed for good focus.

In this paper, we use the electromagnetic particlein-cell code $\mathrm{LSP}^{8,9}$ to investigate the chamber transport of beams in the robust point design. Parameters for the robust point design are reviewed briefly in Section II, and Section III summarizes the results of simulations departing from the point-design parameters in various ways. A concluding section discusses the design space available for more-economical power-plant designs.

\section{POINT-DESIGN PARAMETERS}

The point design builds up the required power profile by using beams with different durations, currents, energies, and arrival times, as sketched in Fig. 1. A total of 120 beams is specified, divided into five classes. The initial lower-energy part of the profile, called the "foot," is built up by stacking three types of beam, and the main pulse is formed from two more types. The large number of beams is needed both to keep the current in each beam manageable and to give each type of beam adequate azimuthal symmetry when deposited in the annuli on the target ends.

The point design specifies beams of singly charged bismuth ions (209 amu). To have the required stopping power, bismuth main-pulse ions require an energy of 4 $\mathrm{GeV}$. Foot-pulse energies are $25 \%$ lower to compensate for the shorter stopping distance at low temperature. The most challenging beams to transport are the initial foot pulses and the final 48 main pulses. These foot pulses are challenging because they must reach the target without additional neutralization from a photoionized plasma around the target, and the 9.3-ns main pulses are singled out because they have the highest current and perveance $^{10}$, which is a measure of the importance of space charge in transverse dynamics. The simulations reported here focus on these two pulse formats. The main pulses have beam current of $2 \mathrm{kA}$ and a perveance of $9 \times 10^{-5}$, and the foot-pulse current is $1.5 \mathrm{kA}$, giving a $7 \times 10^{-5}$ perveance. Both types of pulse are initialized with a normalized transverse edge emittance of $2 \mathrm{~mm}$ $\mathrm{mrad}$, which allows for an increase during acceleration 




Figure 1. Sketch showing how the power profile can be approximated by constant-current beams with different durations. The number of each type of beam is given in parentheses.

and compression of about a factor of five. In all the cases, the beam current falls off in the 3-ns beam ends like a normal ogive, and the radius drops from its midpulse value to $2 \mathrm{~cm}$ with a similar S-shaped pattern.

As discussed elsewhere ${ }^{11}$ in more detail, each beam is focused with a 10-mrad convergence angle onto a target location six meters beyond the last final-focus magnet. A beam first passes through a conical 3-m chamber-entry pipe lined with a molten-salt vortex to protect the surface. This molten-salt layer is modeled as an insulator with a surface conductivity of $220 \mathrm{~S} / \mathrm{m}$. The beam then enters the 3-m radius fusion chamber, filled with $\mathrm{BeF}_{2}$ and $\mathrm{LiF}$ vapor from the molten salt jets used to protect the chamber walls. This background gas has a vapor density of $7 \times 10^{12} \mathrm{~cm}^{-3}$, as is appropriate for thick-liquid walls ${ }^{12}$, and the gas density tapers off to zero in the final $50 \mathrm{~cm}$ of the beam pipe.

Neutralization after final focus is essential for all the cases discussed here. Analytic work by Olson ${ }^{13}$ indicates that the upper perveance limit for ballistic transport is about $1.6 \times 10^{-5}$, so the number of beams would have to be increased more than five-fold to meet this condition. Instead, as in Ref. 11, we neutralize the beam with $10-\mathrm{cm}$ thick layers of plasma, with a density of $3 \times 10^{11} \mathrm{~cm}^{-3}$, near each end of the 3-m entry pipe. Electrons captured by the beam as it passes through this plasma increase the neutralization of the beam space charge to more than $95 \%$ in the chamber, compared with less than $30 \%$ for collisional ionization alone.

\section{RESULTS}

In addition to plasma neutralization, the axisymmetric, single-beam simulations here include electron emission from the entry-pipe and chamber walls, collisional ionization of the beam and background gas, and photoionization by $\mathrm{X}$ rays from the heated target. Details of the numerical model are given in Ref. 11. Other physical processes, such as collisional scattering and electron emission from the target are omitted because they are found to have no significant effect in trial runs.

\section{III.A Effects of Plasma Neutralization}

For the perveance values of the point design, space charge prevents a usable focal spot in the absence of external neutralization. The curves in Fig. 2a show the time variation of the beam root-mean-square (rms) radius at selected axial positions for the 9.3-ns main pulse. The beam minimum radius, or "waist," occurs
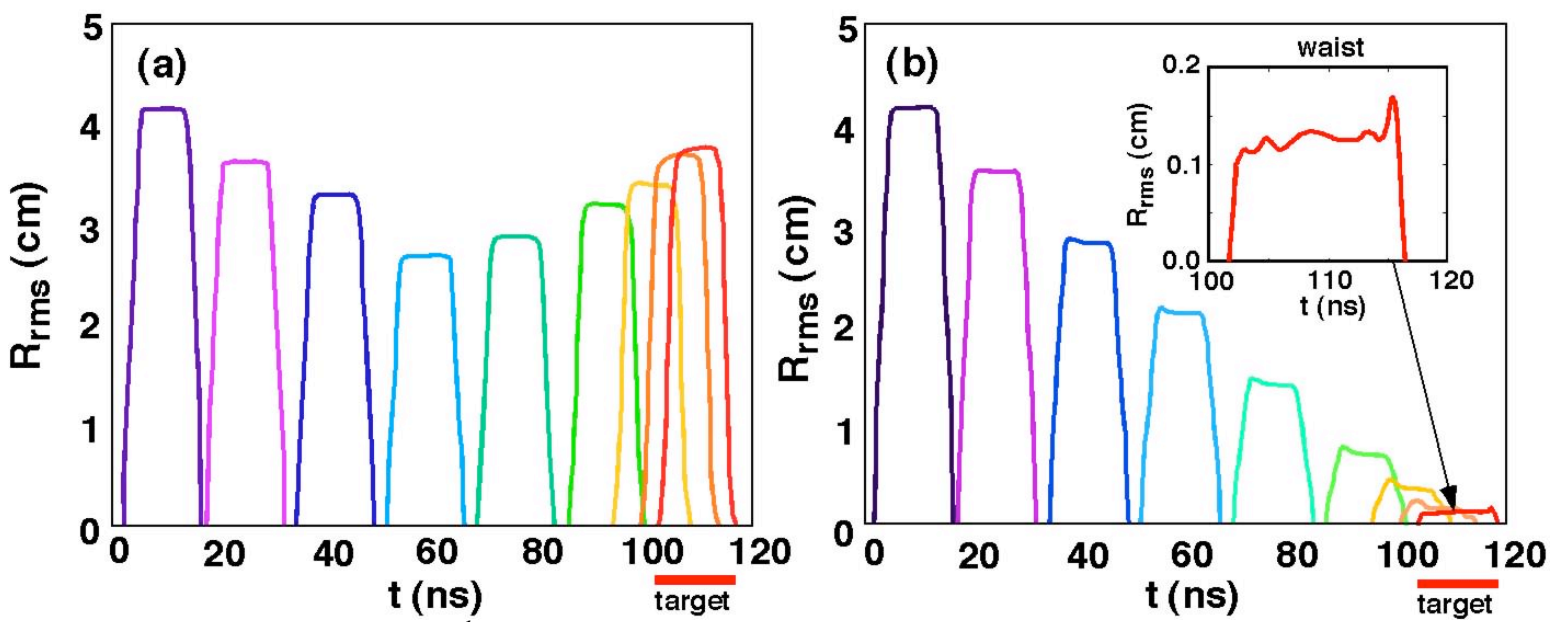

Figure 2. Time variation of a $\mathrm{Bi}^{+1}$ main-pulse rms radius at selected axial locations (a) without plasma neutralization and (b) with plasma placed near the ends of the beam-entry pipe. The time during which the beam traverses the target location is indicated by the red bar beneath each plot. 

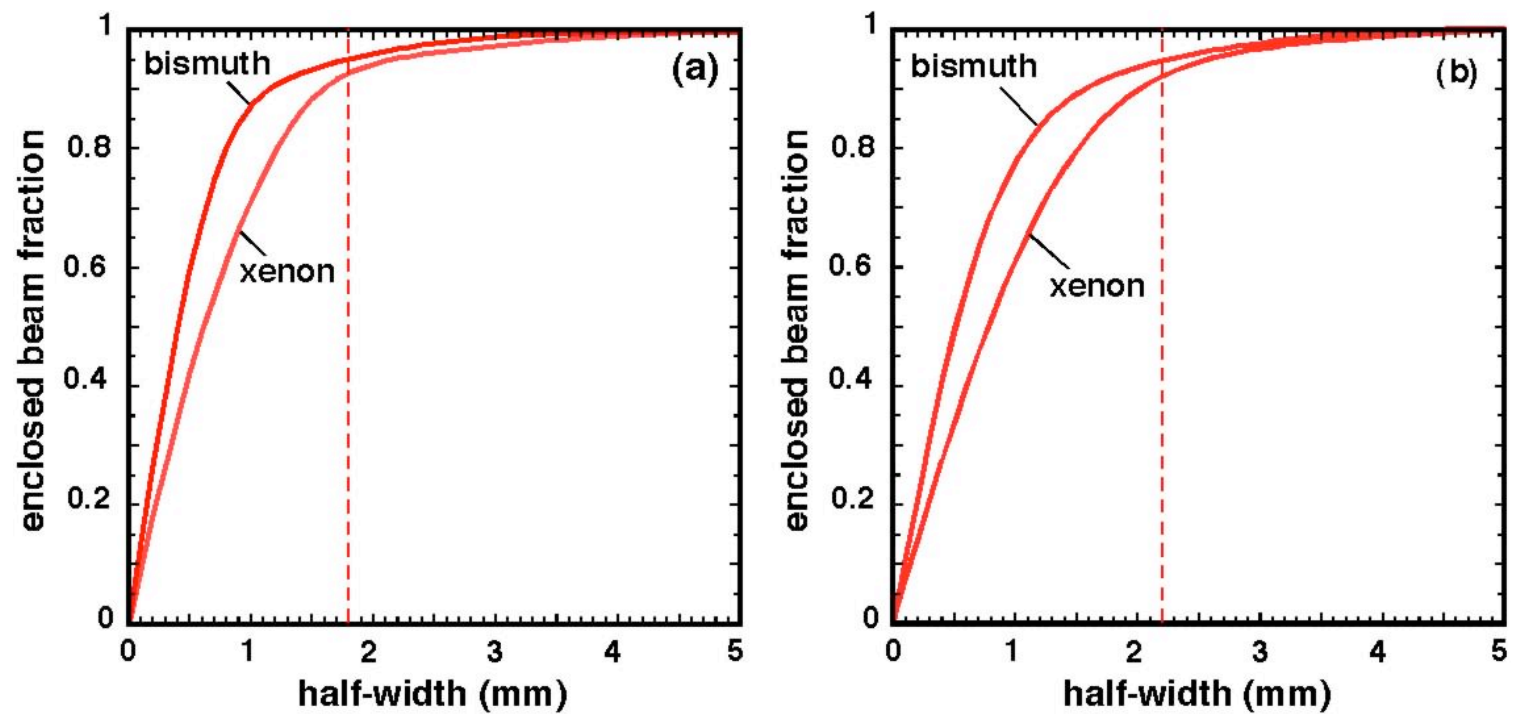

Figure 3. Fraction of beam energy deposited within a band of a given half-width, plotted for xenon and bismuth (a) main pulses and (b) foot pulses. The dashed lines show the respective half-widths of the corresponding target annuli.

after $60 \mathrm{~ns}$ of transport, just after the beam has exited the entry pipe into the chamber, and by the time it reaches the nominal target location, about $100 \mathrm{~ns}$, the radius is larger than the initial value. Although electron emission is allowed from entry-pipe and chamber walls in this simulation, these electrons are ineffective at neutralizing the beam. Emitted electrons are attracted by the beam space-charge field, gaining an energy that can exceed $10^{5} \mathrm{eV}$. Due to this thermal energy, many of these electrons escape the beam as it converges to the target.

For the same main pulse, adding plasma layers in the chamber-entry pipe to neutralize the beam produces the greatly improved focal spot seen in Fig. 2b, with an rms radius of about $1.2 \mathrm{~mm}$ at the waist. In this case, the beam is fully neutralized while it is in each layer, and enough plasma electrons dragged along by the beam as it exits to provide $90 \%$ neutralization of the beam space charge in the entry pipe and more that $95 \%$ in the chamber. An essential aspect of this neutralization technique is that the plasma layers be in electrical contact with a grounded wall, so that electrons can be emitted from the wall to preserve plasma quasineutrality. Without these emitted electrons, spacecharge buildup in plasma limits neutralization of an exiting beam to about $50 \%$. The focal spot in Fig. $2 \mathrm{~b}$ meets the target requirement, but the target physics must still be analyzed to ensure that the ions falling outside the intended annulus do not impair the radiation symmetry.

As foot pulses heat an indirect-drive HIF target, soft $\mathrm{X}$ rays emitted by the hohlraum photoionize the surrounding background gas. For the gas densities expected in liquid-wall chambers, the resulting plasma improves neutralization near the target for main pulses and the late-arriving foot pulses. This additional neutralization is partly offset, however, by photostripping of the beam and by enhanced collisional stripping by the photoionized background gas. Together, these effects lead to a modest improvement in the beam focal spot. If photoionization is artificially turned off in the case with plasma neutralization here, the average charge state drops from about seven to 1.8 , and the focal-spot radius increases by about $10 \%$, even though final emittance is $30 \%$ lower without photoionization. These increases in emittance and charge-state due to photoionization have only a weak effect on the spot size because they occur too close to the target to affect the beam transverse profile.

For the current used here, magnetic self-pinching is negligible, although it is seen in simulations of beams that have initial currents above $4 \mathrm{kA}$.

\section{III.B Effects of Ion Species}

The choice of beam ion species has a major impact on the cost of a driver. Since target stopping power is principally a function of ion velocity, the energy of ions required by a target is approximately proportional to atomic mass $M$. The cost of induction accelerators increases roughly in proportion to the beam energy, so power-plant studies consistently favor low-mass ions. To test the sensitivity of the beam focal radius to ion mass, we compare beams of xenon (131 amu) with the bismuth results. Since beam current increases inversely with $M$ for a given target, the xenon main pulses must have $3.2 \mathrm{kA}$ of $2.5-\mathrm{GeV}$ ions, and foot pulses require 2.4 $\mathrm{kA}$ of $1.9-\mathrm{Gev}$ ions. Beam perveance increases like $M^{2}$, so the corresponding perveance values are $1.7 \times 10^{-4}$ and $2.3 \times 10^{-4}$. To choose a comparable initial emittance for xenon, we note that emittance is roughly proportional to 

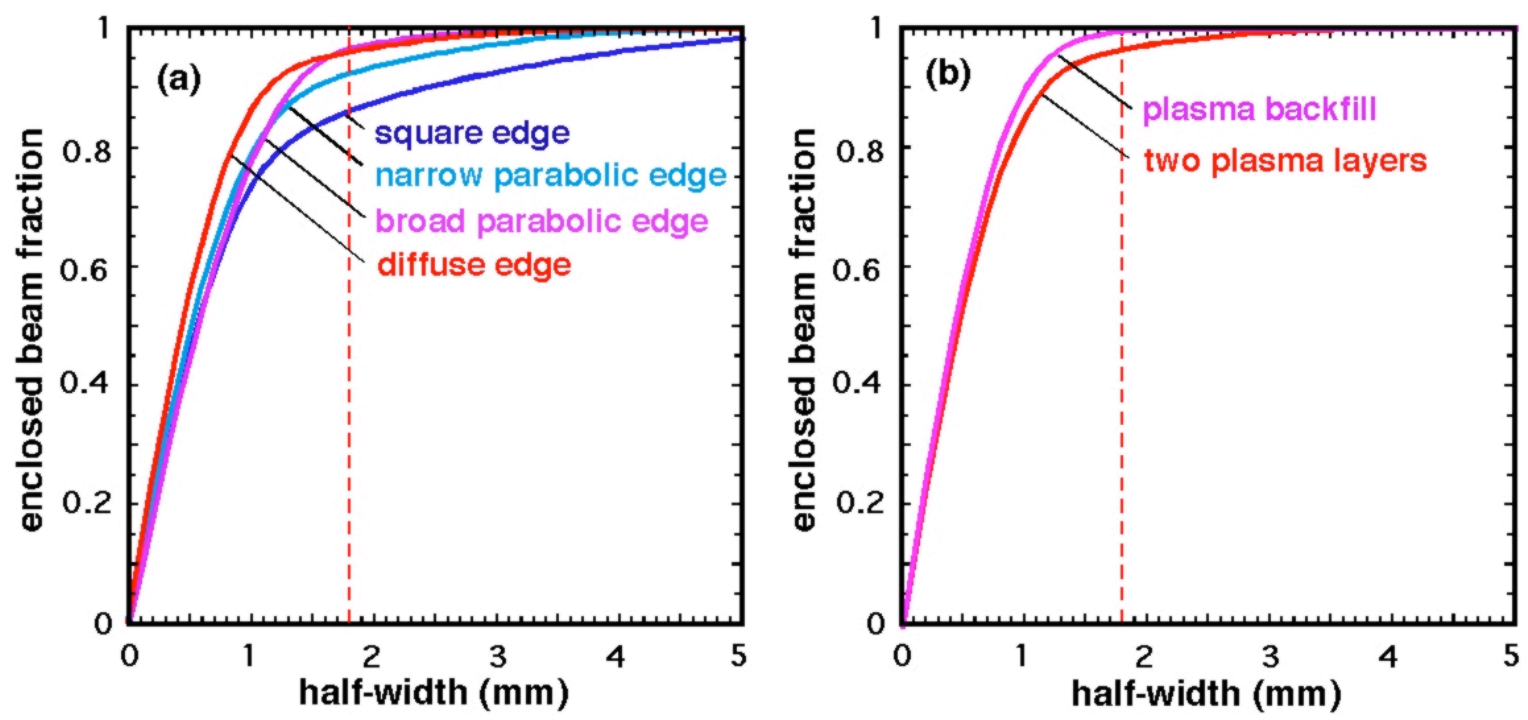

Figure 4. Enclosed beam fractions for bismuth main pulses with (a) various density profiles at the longitudinal edges of the plasma layers, and (b) a plasma backfill in the beam-entry pipe, rather than two plasma layers. The dashed line on each plot shows the half-width of the target annulus for main pulses.

the thermal velocity $(2 k T / M)^{1 / 2}$, where $k$ is the Boltzmann constant and $T$ is the beam temperature. In the cases reported here, we somewhat arbitrarily assume that the initial beam temperatures are the same, so the initial emittance scales like $M^{1 / 2}$.

To make a quantitative comparison of these beam species, we note that distributed-radiator targets require that at least $90 \%$ of the beam energy be deposited in an annulus on each end of the hohlraum. For the specific target used in the point design, the main pulse must hit an annulus with a half-width of $1.8 \mathrm{~mm}$, and the annulus for foot pulses has a 2.2-mm half-width. Therefore, a good criterion for evaluating the simulations is the fraction of energy deposited in a band with a half-width equal to that of the target annulus. This measure ignores the curvature of the annulus but is still a useful approximation.

Figure 3a compares the energy deposition for main pulses of bismuth and xenon. While bismuth has better focus near the center of the pulse and is therefore more robust, as expected, both species satisfy the criterion that at least $90 \%$ of beam energy falls within the target annulus, shown by a dashed line in the figure. The main pulse fractions are $96 \%$ for bismuth and $93 \%$ for xenon.

Due to the absence of a photoionized plasma, the foot pulses for the two species have poorer neutralization near the target, a larger halo, and lower fractional deposition. Nonetheless, the deposition fractions, shown in Fig. 3b, are only about $1 \%$ less than the corresponding main-pulse values, due to the wider target annulus.

\section{III.C Effects of Plasma Parameters}

The calculated beam focal spots are not sensitive to the density of the neutralizing plasma, provided that the number of electrons in the volume swept out by the beam exceeds the total beam charge. The $3 \times 10^{11} \mathrm{~cm}^{-3}$ plasma density used here is about a factor of ten higher than this minimum, but a significant change in the beam focal radius is only seen when the plasma density is lower than about $10^{10} \mathrm{~cm}^{-3}$. A nearby conducting wall enhances plasma neutralization, however, because image charges on the entry-pipe wall alter the plasma space-charge field and makes it easier to remove electrons along the axis. Child-Langmuir electron emission from the wall further improves beam neutralization by keeping the plasma quasi-neutral as electrons are extracted.

Although the beam focal spot is not sensitive to the plasma density, the axial density profile of the first plasma layer encountered by the beam is found to have a pronounced effect. Several cases are shown in Fig. 4a. When the plasma density drops abruptly to zero at the plasma edge, only about $85 \%$ of the energy of a bismuth main pulse falls within the requisite $1.8-\mathrm{mm}$ half-width band, but this fraction increases to $92 \%$ when the density falls parabolically over $3-\mathrm{cm}$ and to $97 \%$ for a 6 $\mathrm{cm}$ parabolic or normal-ogive edge. An examination of these cases shows that a current of back-streaming electrons nearly equalling the beam current develops as the unneutralized beam approaches the square-profile plasma. This current flows near the beam axis, and the resulting nonlinear space-charge field within the beam causes a substantial emittance increase. Both the electron current and the emittance increase are less for the other plasma profiles. For example, we see only a $10 \%$ emittance increase in the entry pipe for the ogive profile, compared with nearly a doubling for a square plasma edge. We find in this case that electrons from the low-density upstream edge of the first plasma layer reduce the beam space-charge field as the beam approaches, and the more-gradual neutralization that 

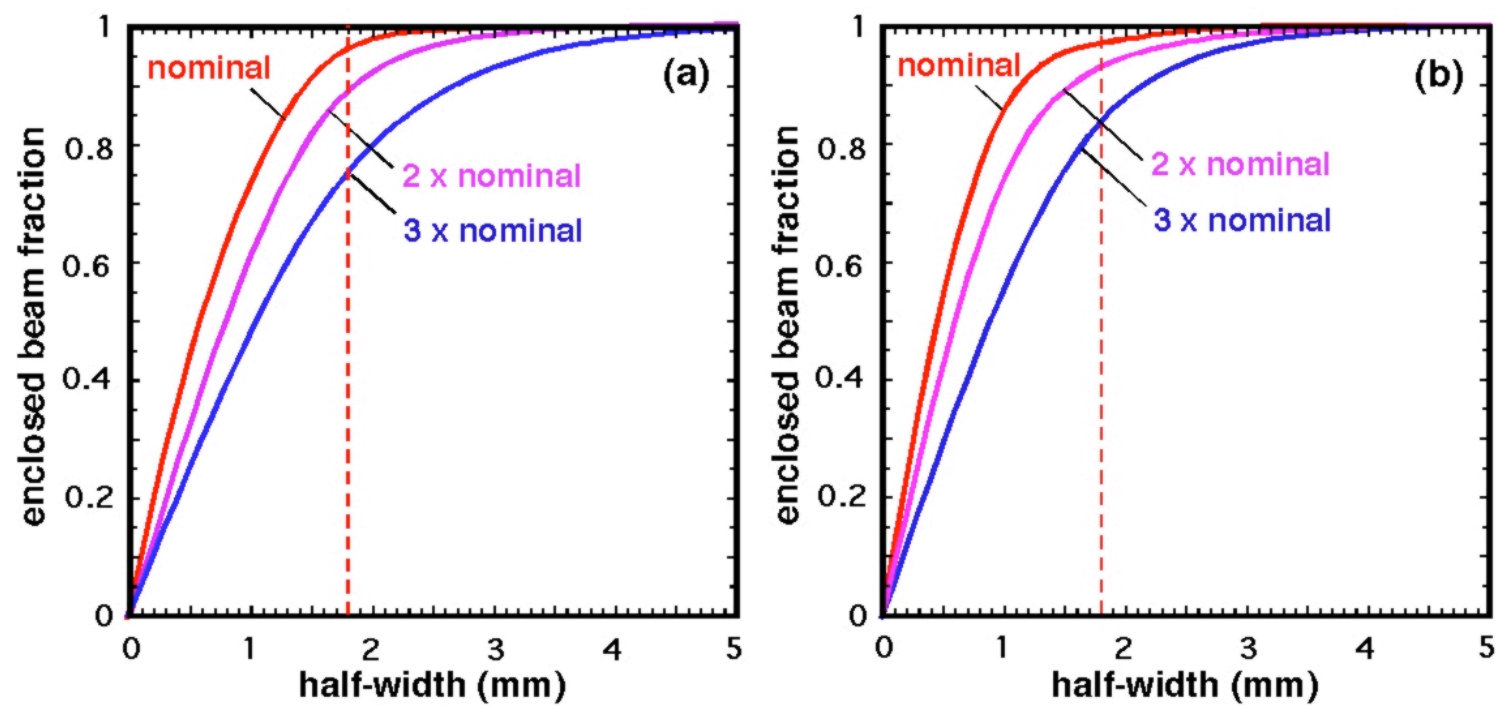

Figure 5. Enclosed beam fractions for (a) xenon and (b) bismuth main pulses with the twice and three times the nominal initial emittance. For comparison, the corresponding values for the nominal emittance are replotted from Fig. 3a. The dashed line on each plot shows the half-width of the target annulus.

results leads to the smaller emittance growth. For the remaining cases here, we use the more realistic ogival edge profile.

Sensitivity of the beam focal spot to the length of the neutralizing plasma has also been studied. Specifically, we have compared placing a plasma layer near each end of the entry pipe against backfilling the entire entry pipe with plasma. In both cases, each plasma end is modeled by a $6-\mathrm{cm}$ ogival profile, and the same $3 \times 10^{11} \mathrm{~cm}^{-3}$ interior density is specified. As Fig. $4 \mathrm{~b}$ shows, a plasma backfill increases the energy deposited on the target annulus by about 3\% for both bismuth and xenon main pulses. Nonetheless, we use the layout with two plasma layers for other cases here because it poses fewer engineering problems.

\section{III.D Effects of Initial Emittance}

The nominal $2 \mathrm{~mm}$-mrad initial emittance used here puts a stringent constraint on emittance growth from source through final focus. The simulations shown in Fig. 5 predict a marked degradation in the beam focal spot when this condition is not met. When the initial emittance of a xenon main pulse is doubled, for example, the energy deposited in a 1.8 - $\mathrm{mm}$ half-width band drops from $96 \%$ to $90 \%$, and this fraction drops to about $85 \%$ when the emittance is tripled. Bismuth beams are somewhat less sensitive to emittance change, but a tripling of the nominal value still leads to an unusable focal spot. Since the emittance grows only about $20 \%$ in the chamber when a realistic plasmadensity profile is used, emittance growth in the accelerator is tightly constrained.

\section{III.E Effects of Convergence Angle}

The beam convergence angle of a beam, given by the initial edge radius divided by the focal distance, should be as small as possible in a driver design for several reasons. The unshielded solid angle around the target through with neutrons, gamma rays, and debris can escape is proportional to the square of the beam radius, so reducing the convergence angle simplifies shielding. In addition, the bundle of beams converging on each end of the hohlraum can have a smaller cone angle, allowing the use of higher-gain targets as well as smaller and less costly final-focus magnets.

To assess how energy deposition on the target varies with the convergence angle, we need some model of how the transverse emittance after final focus varies with the beam radius. When emittance growth during chamber transport is ignored, an envelope equation ${ }^{14}$ for the beam rms radius predicts that the beam radius at the waist is given roughly by the transverse emittance divided by the convergence angle. We therefore assume that emittance remains constant as the radius changes, since this choice should show any sensitivity to the convergence angle with the least ambiguity. The nominal convergence angle for the robust point design is $10 \mathrm{mrad}$, and we have run a series of bismuth and xenon cases with this angle decreased up to a factor of two. As Fig. 6 shows, both species show less than a $4 \%$ drop in the energy deposited within the target annulus for a $7.5-\mathrm{mrad}$ convergence angle, with the fraction of energy deposited there remaining comfortably above $90 \%$. Deposition, however, drops to unacceptable levels when the convergence angle is decreased to $5 \mathrm{mrad}$. In all these cases, the axial location of waist, nominally 6 

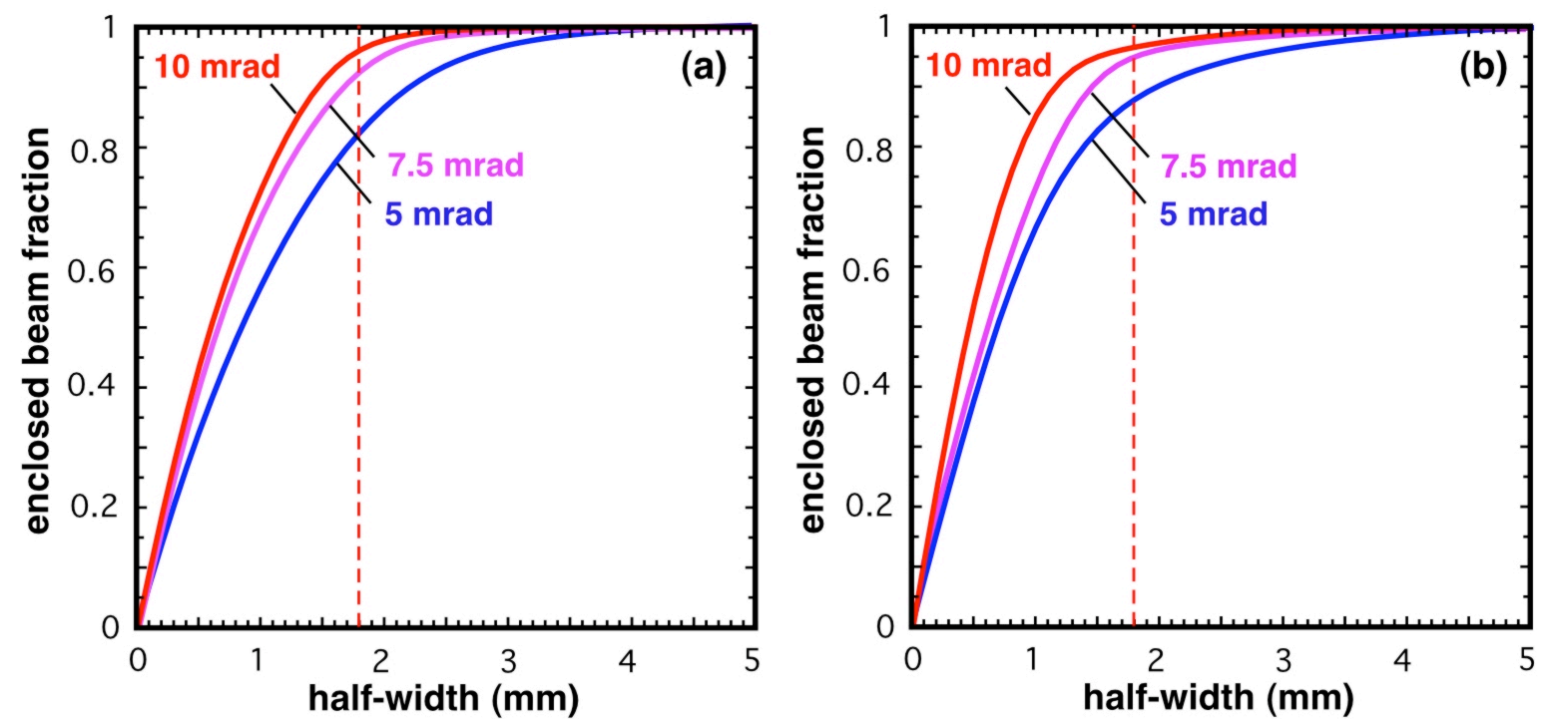

Figure 6. Enclosed beam fractions for (a) xenon and (b) bismuth main pulses with the nominal 10-mrad convergence angle, as shown in Fig. 3a, and with two smaller angles. The dashed line on each plot shows the halfwidth of the target annulus.

$\mathrm{m}$ from the final magnets is found to increase as the convergence angle is reduced. For both species. the waist occurs at about $6.1 \mathrm{~m}$ for a 7.5 -mrad convergence angle and at $6.25 \mathrm{~m}$ for $5 \mathrm{mrad}$. This focal-point error, however, is easily corrected without significantly affecting the waist radius by refocusing the beam to a point shifted forward from the nominal waist by the amount of the error. These simulations suggest that the nominal 10-mrad convergence angle of the robust point design can be reduced by up to $25 \%$ with little degradation of the focal spot.

\section{III.F Effects of Initial Charge State}

Recent work on beam sources indicates that intense beams of negatively charged halogen ions may be feasible. ${ }^{15}$ Such beams are attractive as HIF drivers because they avoid the electron-cloud effects possible with positive beams. ${ }^{16,17}$ Due to the low binding energy of the outer electron $(\sim 3 \mathrm{eV})$, such beams could be photoneutralized after final focus by a $\mathrm{KrF}$ or xenon laser of moderate intensity ${ }^{18}$, so they would not need plasma neutralization.

We have completed preliminary chamber-transport simulations photoneutralized halogen beams. Assuming that the beams can be photoneutralized over a few centimetres, as estimated in Ref. 17, the change in transverse energy due to space charge over this distance should be a small fraction of the directed radial kinetic energy, allowing us to treat the beam as initially neutral. For main pulses of iodine (127 amu), bromine (80 amu), and chlorine (35 amu), parameters are chosen to give the same beam power and stopping distance as the point design. The particle current is $3.3 \mathrm{kA}$ for the iodine beam, $5.25 \mathrm{kA}$ for bromine, and $11.9 \mathrm{kA}$ for chlorine, and the respective energies are $2.4 \mathrm{GeV}, 1.5 \mathrm{GeV}$, and $0.68 \mathrm{GeV}$. For comparison, we have also simulated a plasma-neutralized $\mathrm{I}^{+1}$ beam and a $\mathrm{I}^{-1}$ beam without plasma neutralization. The results are shown in Fig. 7. Due to the long mean-free path for stripping in the chamber at the assumed $7 \times 10^{12} \mathrm{~cm}^{-3}$ gas density, both the mean charge state and the net charge of the initially neutral iodine and bromine beams remain less then corresponding values for the plasma-neutralized $\mathrm{I}^{+1}$ beam. Consequently, the fraction of energy deposited in a $1.8-\mathrm{mm}$ half-width band exceeds $98 \%$ for both beams, compared with $93 \%$ for the $\mathrm{I}^{+1}$ with a plasma. The fraction is about $92 \%$ for the chlorine beam, due to its higher net charge, but the halo extends to a smaller radius than that of the $\mathrm{I}^{+1}$ beam. The unneutralized $\mathrm{I}^{-1}$ beam degrades in the 3-m entry pipe due to its own space charge, so it reaches a waist before being stripped by collisional ionization in the chamber and expands to a large radius by the time it reaches the target location.

To corroborate these results, further simulations are needed in which initially negative ions are neutralized over a plausible time and distance in the entry pipe. If the results remain favorable, this improved beam deposition might permit a simpler chamber design due the absence of plasma neutralization and allow the use of beams with either higher emittance or substantially lower energy.

\section{Conclusions}

The beam and chamber parameters of the point design studied here reconcile the conflicting demands of accelerator cost, neutronics, final-focus, and target design for a HIF driver. The final choice of 120 beams of singly charged bismuth at $3-4 \mathrm{GeV}$ is in no sense 


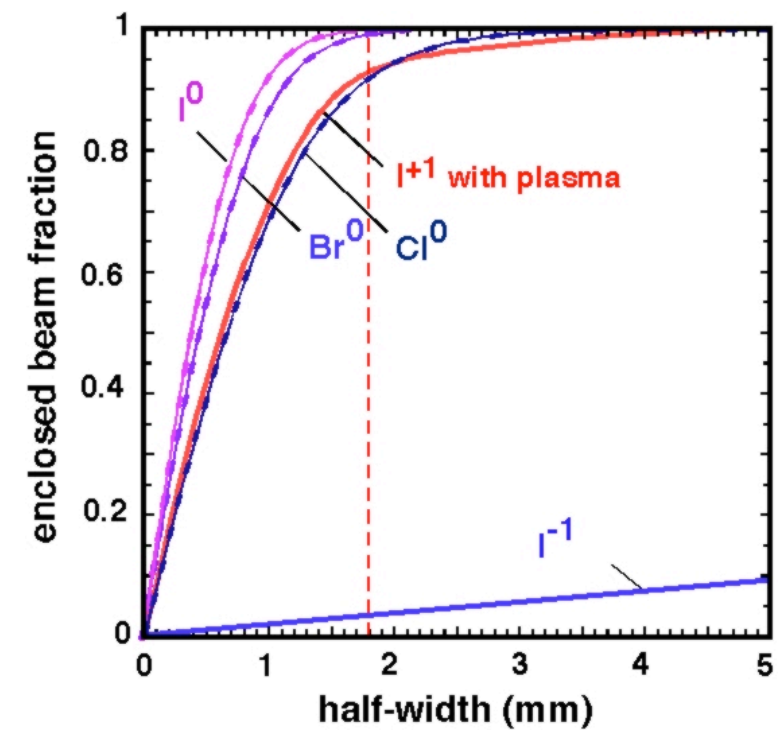

Figure 7. Enclosed beam fractions for initially neutral iodine, bromine, and chlorine main pulses without neutralization. For comparison, the corresponding curves are also shown for a plasmaneutralized $I^{+1}$ beam and for an $I^{1}$ beam without plasma neutralization. The dashed line shows the target-annulus half-width.

optimum, but the simulation results to date indicate that such beams could be successfully transported to a fusion target and meet the requirements on spot size and deposition history. A crucial aspect of the successful transport is the use of low-density plasma to neutralize beams before they enter the chamber.

The simulations show some flexibility in the pointdesign parameters. The use of ions with masses as low as that of xenon appears feasible, and the beam convergence angle can be reduced by $25 \%$ without seriously compromising the focal-spot size. Both changes might reduce the cost and complexity of a driver. The focal-spot radius is found to be sensitive initial beam emittance, which can be at most doubled before the focal spot becomes unusable. Backfilling the chamber-entry pipe rather than injecting plasma layers near the two ends reduces the beam net charge and leads to a smaller focal spot, possibly allowing the use of lower-mass ions or higher emittance at the cost of greater complexity. Some further relaxation of beam requirements might be possible if negative-ion beams of sufficient current can be accelerated and then photoneutralized after final focus.

In future simulations, we will add several features of chamber physics to the numerical model, particularly some representation of the molten-salt jets, and we will begin using more realistic distribution functions for the input beam. At the same time, the collaboration will continue between researchers working on the different accelerator subsystems to produce a workable physics design.

\section{Acknowledgments}

This work was performed under the auspices of the US Department of Energy by University of California Lawrence Livermore National Laboratory and Lawrence Berkeley National Laboratory under Contracts No. W7405-ENG-48 and DE-AC-3-76SF00098.

\section{REFERENCE}

${ }^{1}$ LINDL, J., Phys. Plasmas 2, 3933 (1995).

2 TABAK, M., CALLAHAN, D. A., Nucl. Instr. and Meth in Phys. Res. A 415, 75 (1998).

3 CAllahan-Miller, D. A., TABAK, M., Nucl. Fusion 39, 1547 (1999).

${ }^{4}$ YU, S. S., et al., "An Updated Point Design for Heavy Ion Fusion," Lawrence Livermore National Laboratory Report UCRL-JC-150169, (2002).

5 ROSE, D. V., et al., Nucl. Instr. and Meth in Phys. Res. A 464, 299 (2001).

${ }^{6}$ WELCH, D. R., et al., Phys. Plasmas 9, 2344 (2002).

${ }^{7}$ SHARP, W. M., et al., Phys. Plasmas 10, 2457 (2003).

${ }^{8}$ HUGHES, T. P., et al., Phys. Rev. ST Accel. Beams 2, 110401 (1999).

${ }^{9}$ WELCH, D. R., et al., Nucl. Instrum. Meth. Phys. Res A 464, 134 (2001).

${ }^{10}$ LAWSON, J. D., J. Electron. Control 5, 146 (1958).

11 SHARP, W. M., et al., Fusion Sci Tech. 43, 393 (2003).

12 OLANDER, D. R., et al., Fusion Sci. Tech. 41, 141 (2002).

${ }^{13}$ OLSON, C. L., Nucl. Instr. and Meth in Phys. Res. A 464, 118 (2001).

${ }^{14}$ KAPCHINSKY, I. M., VLADIMIRSKY, V. V., Proc. International Conference on High Energy Accelerators, (CERN, Geneva, 1959), p. 274ff.

${ }^{15}$ GRISHAM, L. R., "Proof-of-Concept Experiment for Negative Ion Driver Beams for Heavy Ion Fusion," Proc. 2003 Part. Accel. Conf., 12-16 May 2003, Portland, OR, p. 3311.

${ }^{16}$ COHEN, R. H., et al., "Stray-Electron Accumulation and Effects in HIF Accelerators," Proc. 2003 Part. Accel. Conf., 12-16 May 2003, Portland, OR, p. 132.

${ }^{17}$ MOLVIK, A. W., et al., "Initial Experimental Studies of Electron Accumulation in a Heavy-Ion Beam," Proc. 2003 Part. Accel. Conf., 12-16 May 2003, Portland, OR, p. 312.

${ }^{18}$ GRISHAM, L. R., "Evaluation of Negative Ion Beam Driver Concepts for Heavy Ion Fusion," Fusion Science and Technology 43, 191 (2003). 